Thorax (1965), 20, 153.

\title{
Diffuse interstitial pulmonary fibrosis in a mother and son with von Recklinghausen's disease
}

\author{
R. IS R A EL-A S SELAIN, J C CHEBAT, CH. SOR S, \\ F. BASSET, ANDA. LE ROLLAND \\ From Hopital Foch, Suresnes, Paris
}

Specific thoracic manifestations of von Recklinghausen's disease (multiple neurofibromatosis) have been recognized for a long time. They occur in about $15 \%$ of all cases of the disease (Schmitt, 1959). Most of them are neuromatous tumours arising in the mediastinum from the sympathetic chain, the vagus or intercostal nerves. Rarely, either single or multiple subpleural, bronchial or pulmonary neurofibromata may occur. Generally these are benign lesions, but about $10 \%$ undergo malignant change, sometimes after surgical interference. All these lesions have undoubted histological specificity. The literature makes no mention of non-specific diffuse interstitial fibrosis in von Recklinghausen's disease such as was found in the two cases reported here.

\section{CASE REPORTS}

CASE 1 Mrs. J.C. had cutaneous neurofibromatosis with many pigmented areas and pendulous skin tumours. There was a strong family history of the disease; three of her brothers had identical lesions and so has her son, the second patient, and her grandson.

In 1954, when she was 64 years, she complained of increasing dyspnoea. At that time the chest radiograph showed pleural shadowing in both upper zones, more marked on the right than the left, and diffuse linear and reticular opacities throughout the lung fields, most marked in the upper lung fields (Fig. 1).

In spite of negative sputum cultures for tubercle bacilli, a diagnosis of pulmonary tuberculosis was made, and she was treated for several months with streptomycin and P.A.S. but without change in the radiological appearances.

In 1957 her dyspnoea increased and she developed a dry cough. Her general condition remained unchanged and she had no fever. Radiologically the appearances had deteriorated. There was an increase both of the pleural opacity and of the coarse linear opacities in the lung fields. Sputum cultures were again negative for tubercle bacilli, and further antituberculous treatment with streptomycin and I.N.A.H. was again ineffective.
In 1959 her general condition deteriorated considerably. Her dyspnoea was severe and she had a constant cough productive of mucopurulent sputum but without haemoptysis. Radiologically there was also considerable deterioration. The pleural shadowing had increased; there were reticulo-nodular opacities throughout the lung fields (Fig. 2) and an irregular cavity had appeared in the right upper zone.

No treatment was of any avail, and she died of respiratory failure in January 1960.

Pathological anatomy The right lung showed pleural thickening, especially on the posterior and apical surfaces. The upper lobe was partially collapsed ; on palpation it felt hard, unlike the middle and lower lobes which had a normal consistency.

On section of the lung in the plane of the main bronchus a vast brownish irregular cavity, typical of the appearances of an aspergilloma, was revealed in the upper lobe. The remainder of this lobe was made up of dense non-aerated tissue, firm under pressure, crossed by thick white strands. The lower edge of the upper lobe was formed by a white sclerotic band $0.5 \mathrm{~cm}$. thick, extending from the thickened pleura at the periphery. The structure of the parenchyma of the middle and lower lobes was less dense and had a spongy appearance.

The parenchyma was condensed by a tissue fibrosis interspersed with microcysts giving the appearance of a honeycomb lung.

In many places the alveoli were entirely replaced by fibrous tissue. In other places the alveolar walls were thickened by collagen and dystrophic elastic fibres together with hyperplastic smooth muscle and, in some areas, dystrophic nerve fibres (Fig. 3).

Both pulmonary and bronchial arteries and their many anastomoses showed thromboses and recanalization. Some of the vessels showed a thickening of their muscle coats containing many nerve fibres giving them an appearance resembling that of glomi ; these vessels were particularly prominent in the walls of dilated bronchi, where they were surrounded by numerous large nerve trunks. The anastomosing bronchial arteries had thick hyalinized walls.

In some areas the bronchioles were dilated, in others they were obliterated. In general, the bronchi were 


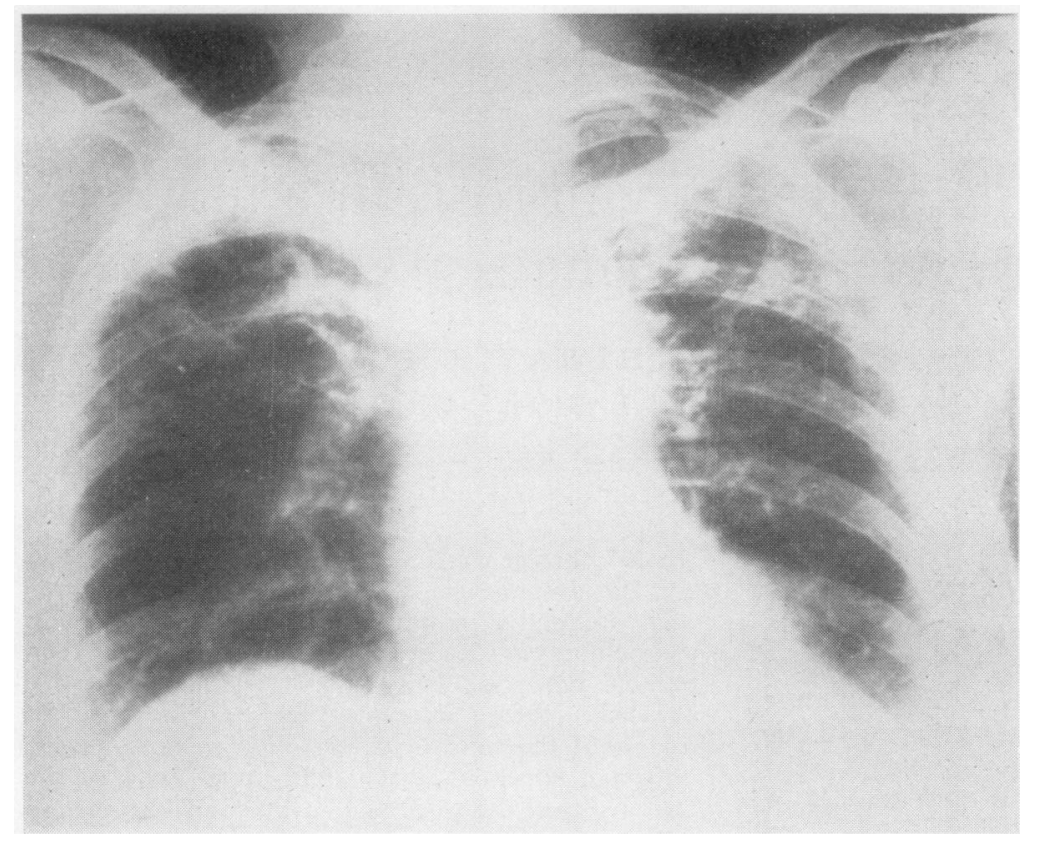

FIG. 1. Case 1. Chest radio $\stackrel{-}{\circ}$ graph 1954. Pleural shadowing $\vec{\omega}$ both upper zones and diffuse irregular linear opacities in both $\overrightarrow{\overrightarrow{\times}}$ upper zones.

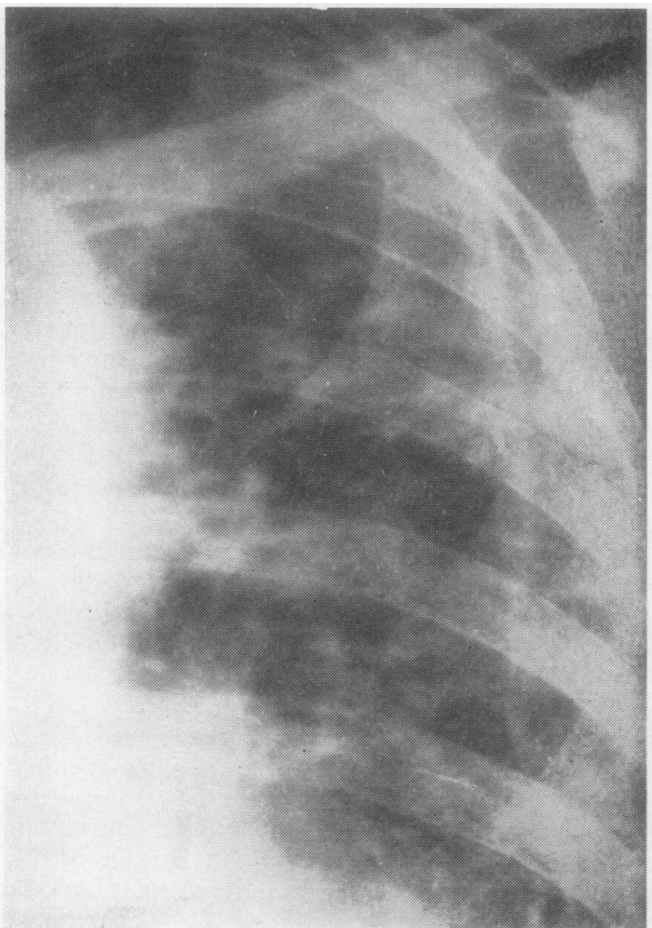

FIG. 2. Case 1. Chest radiograph 1959. Enlargement of left mid and upper zones showing well-marked linear and patchy shadowing in the lung field.

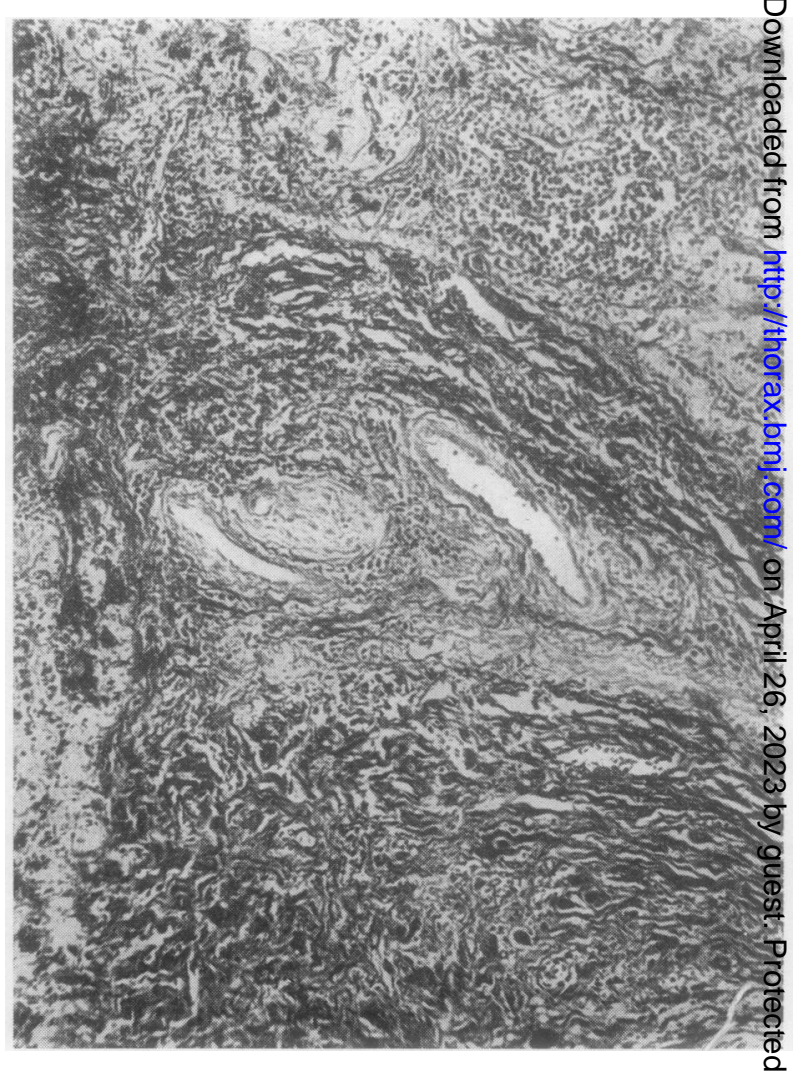

FIG. 3. Case 1. Extensive hyaline fibrosis. Nerve fibril, 무 close to a blood vessel, prominent in the centre of t'ie field. Van Gieson, $\times 110$. 


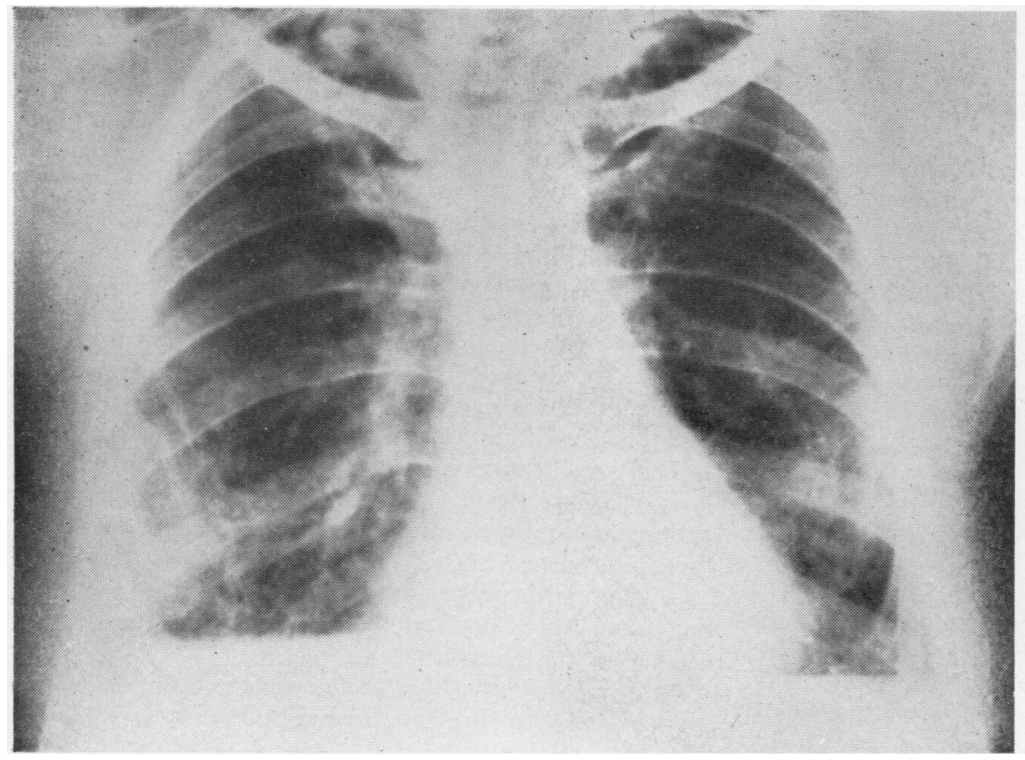

FIG. 4. Case 2. Chest radiograph

1960. Pleural shadowing and diffuse nodular opacities, most prominent in the lower zones.

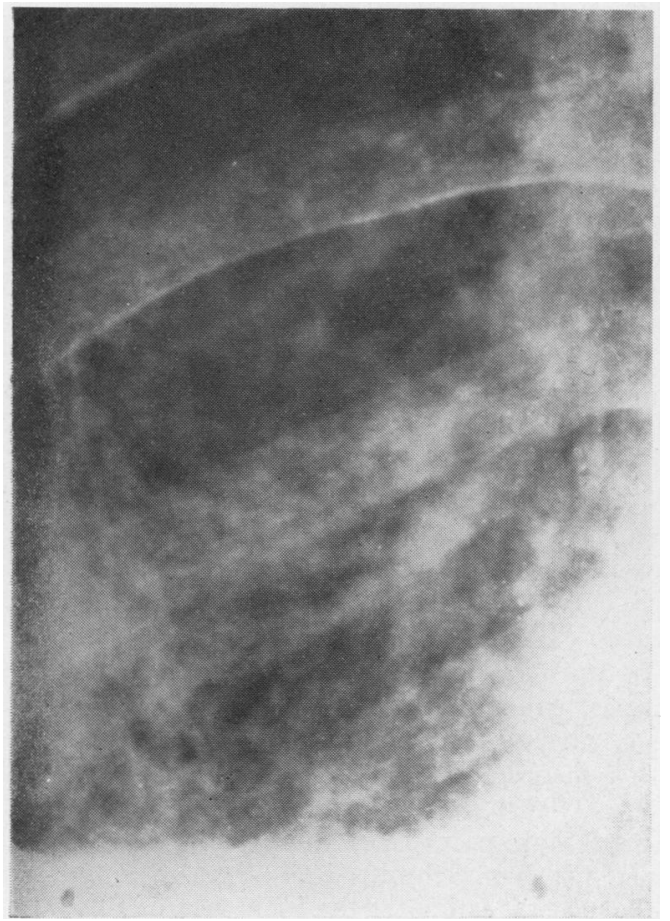

FIG. 5. Case 2. Chest radiograph 1960. Enlargement of right lower zone showing linear pleural shadowing and nodular opacities in the lung field.

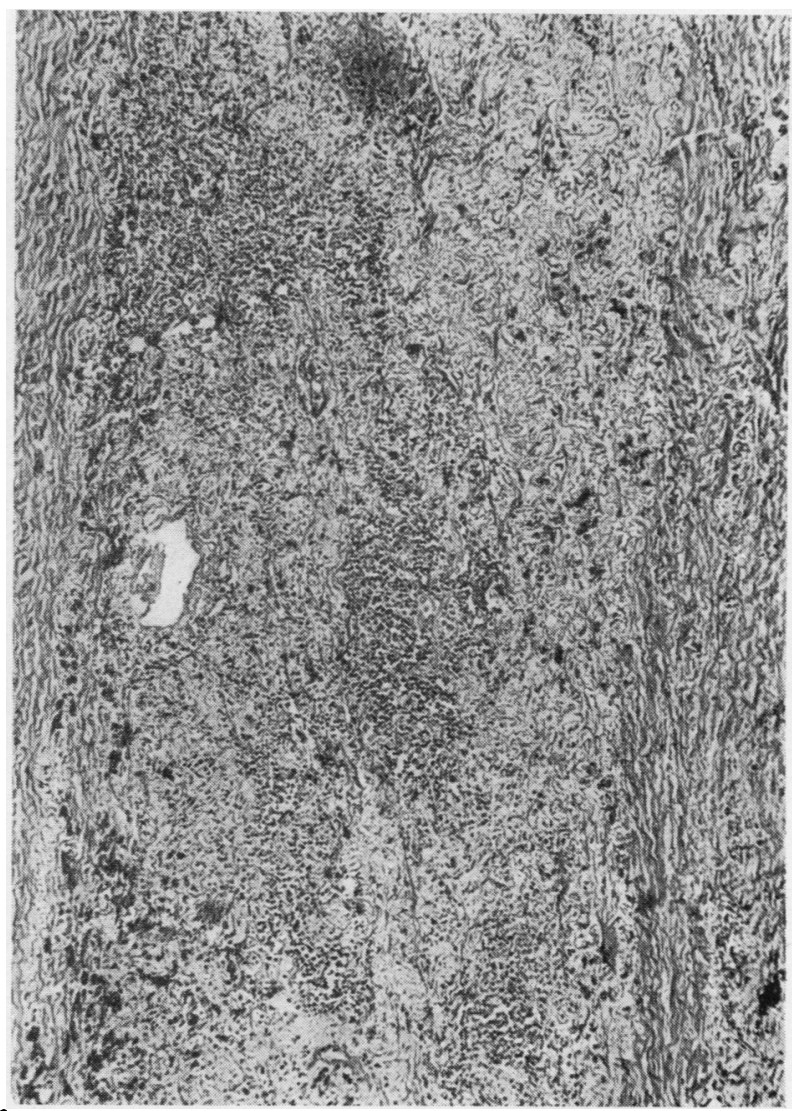

FIG. 6. Case 2. Lung biopsy. Uniform sub-pleural hyaline fibrosis. Van Gieson, $\times 90$. 
dilated and showed marked degeneration of their cartilage.

In summary, the most striking abnormalities were (1) a diffuse destructive pulmonary fibrosis; (2) a marked elastic tissue and leiomyomatous dystrophy: (3) vascular changes of an unusual type; and (4) dystrophic nerve fibres and hyperplastic nerve trunks.

The wall of the aspergilloma was composed largely of hyalinized fibrous tissue with some granulomatous and highly vascularized areas of congestion infiltrated with plasma cells and mycelial filaments.

CASE 2 Mr. M. C. is the son of the first patient. He has a mentally defective son aged 10 , who has von Recklinghausen's disease but whose chest radiograph shows no abnormality.

The superficial signs of neurofibromatosis are areas of skin pigmentation, cutaneous and subcutaneous tumours, numerous small cutaneous angiomas, and craniofacial hemihypertrophy. The diagnosis has been confirmed by biopsy of a skin tumour.

This patient was admitted to hospital in June 1960 at the age of 38 for the investigation of respiratory symptoms and radiographic abnormalities which had appeared during an influenza-like illness in April 1960. He had never had a chest radiograph taken before that time. He complained of slight effort dyspnoea and dry morning cough. There were no abnormal physical signs in the respiratory system and there was no clubbing.

Chest films and tomograms showed unusual abnormalities (Fig. 4). The diaphragms were flat and the costophrenic angles obliterated. There was bilateral symmetrical pleural shadowing which extended obliquely into the lung fields in the midzones. Throughout the lung fields there was diffuse fine reticulo-nodulation (Fig. 5). On fluoroscopy the movements of the diaphragm were unexpectedly normal, the range of movement on both sides being about $5 \mathrm{~cm}$. Bronchoscopy showed no abnormality.

Respiratory function studies The lung volume was reduced; the vital capacity was $30 \%$ below the predicted normal. There was no evidence of air trapping. the ratio of the residual volume to the total lung capacity being $24 \%$. There was no evidence of airway obstruction, the ratio of the forced expiratory volume in one second (F.E.V.1) to the forced vital capacity (F.V.C.) being $77 \%$ and the air velocity index near to 1. There was no evidence of disturbance of gas transfer; the minute volume at rest was $6,480 \mathrm{ml}$.; the respiratory equivalent was 281 , the arterial $\mathrm{PCO}_{2}$ was $34 \mathrm{~mm}$. $\mathrm{Hg}$ and the arterial oxygen saturation was $97 \%$ at rest with only slight desaturation on exertion. The diffusing capacity measured with carbon monoxide by the method of inspiratory apnoea was within the normal range ( $D_{\text {Lc }}, 16.5 \mathrm{ml} . / \mathrm{min} . / \mathrm{mm} . \mathrm{Hg}$ ).

Histological studies A lung biopsy showed the pleura to be adherent.
In the pleura there was a lamellar hyaline sclerosis which was rather acellular but contained nerve fibres and was richly vascularized.

The parenchyma of the lung was rather collapsed (Fig. 6). The septum was thickened by fibrosis with prominent layers of smooth muscle, dilated capillaries, and blood vessels with thickened walls. There was a marked dystrophy of elastic tissue within the collagen $\overrightarrow{0}$ sclerosis which thickened the alveolar walls, and bundles of dystrophic nerve fibres could be seen.

The epithelium of the bronchioles was unchanged but the fibrosis involved their walls.

The appearance of the blood vessels was particu- iv larly striking. Besides the many capillaries, arteries of iv bronchial type were numerous in the thickened septa $; \vec{G}$ they had something of the appearance of the vessels $\omega$ in a glomus, having prominent muscle coats contain- 음 ing nerve fibres derived from large nerve trunks with thickened perineurium.

On the whole the lesions derived from the thickened pleura and the directly subjacent interlobular septa. The unusual features of this pulmonary fibrosis were the vascularization of the interlobular septa, the $\mathscr{C}$ hyperplasia of the nerve trunks, and the relative extent $G$ of the leiomyomatous and elastic tissue dystrophy.

\section{DISCUSSION}

Two patients, a mother and son, suffering from von Recklinghausen's disease (multiple neurofibromatosis), have been found to have diffuse interstitial pulmonary fibrosis with the typical histological characteristics of that condition in its chronic form. The essential features are localization of fibrosis in the interalveolar septa, the presence of leiomyomatous zones, and marked hyperplastic dystrophy of the elastic fibres as well as distinctive vascular changes. The changes are at an early stage in the son aged 38 but were far advanced in the mother, who died as the result of pulmonary involvement at the age of 70 years.

Although interstitial pulmonary fibrosis has not previously been reported in von Recklinghausen's disease, it is well known in other related diseases which are also hereditary, the neurocutaneous $N$ diseases called in France the 'phacomatoses'.

The most common of these diseases is tuberous sclerosis, called in France 'Bourneville's disease'. The occurrence of interstitial pulmonary fibrosis in this disorder was first reported by Berg and Vejlens (1939) in two sisters. In 1954 Dawson $\mathbb{E}$ collected eight cases from the literature and added four of his own. Schmitt (1959), in a review of the whole subject, estimates the incidence of this complication at about $9 \%$ of cases.

The clinical, radiological, and pathological features of the pulmonary lesions in tuberous sclerosis are the same as those of other chronic 
interstitial pulmonary fibroses, honeycomb lung, though it has been argued that the degree of leiomyomatosis is special to the disease ; this is discussed in detail by Roujeau and Morel (1961) in a case report.

The second disease of the same group in which interstitial pulmonary fibrosis has been found is angiomatosis cerebri associated with trigeminal naevus, Sturge-Weber's disease. Schmitt, in his thesis (1959) reports a case in which an interstitial pulmonary fibrosis was discovered at necropsy and in which leiomyomatosis was an important feature.

The finding in one family of two cases of interstitial fibrosis in von Recklinghausen's disease is yet one more manifestation of the many similarities that have been shown between three diseases, von Recklinghausen's disease, tuberous sclerosis, and Sturge-Weber's disease. All three are hereditary diseases affecting the neuro-ectodermal tissues (skin and nervous system). The term 'phacomatosis' was introduced by Van der Hoeve (1933) to symbolize this relationship (Phakos is Greek for 'spot'). Since then many synonyms have been employed; the latest, neuro-ectomesodermatosis
(Kissel, Dureux, and Schmitt, 1962), has the merit of drawing attention to the involvement of mesoderm in this group of diseases, thus serving to stress the possibility of pulmonary changes.

\section{SUMMARY}

Two cases of diffuse interstitial pulmonary fibrosis in a mother and son with von Recklinghausen's disease (multiple neurofibromatosis) are described.

We are grateful to Dr. P. D. B. Davies for translating our paper into English.

\section{REFERENCES}

Berg, G., and Vejlens, G. (1939). Maladie kystique du poumon et sclérose tubéreuse du cerveau. Acta paediat. (UI $\left.f s c l_{6}\right), 26,16$. Dawson, J. (1954). Pulmonary tuberous sclerosis. Quart. J. Med., 23, 113.

Kissel, P., Dureux, J. B., and Schmitt, J. (1962). I es Fhaccmatoses. Considérations générales. Intérêt spéculatif et pratique. Rev. Prat. (Paris), 12, 915.

Roujeau, J., and Morel, R. (1961). Les localisations pulmcnaires de la sclérose tubéreuse de Bourneville. J. frarc. M'́d. Chir. thor., $15,247$.

Schmitt, J. (1959). Les Formes Viscérales des Phacomatoses. Doin, Paris.

Van der Hoeve, J. (1933). Les phakomatoses de Bourneville, de Recklinghausen et de von Hippel-Lindau. J. belge Neurol. Psychiat., 33, 752. 